\title{
Isopsoralen ameliorates rheumatoid arthritis by targeting MIF
}

\author{
Yi Han', Jinguang Wang ${ }^{2}$, Shufeng $\mathrm{Li}^{3}$, Yi Li ${ }^{4}$, Yongli Zhang ${ }^{5}$, Ruojia Zhang ${ }^{5}$, Yuang Zhang ${ }^{5,6,7}$, Huancai Fan ${ }^{5}$, \\ Haojun Shi ${ }^{8}$, Jihong Pan ${ }^{5,6,7}$, Guanhua Song ${ }^{9}$, Luna Ge $\mathrm{G}^{5,6,7^{*}}$ and Lin Wang ${ }^{5,6,7^{*}}$
}

\begin{abstract}
Background: Isopsoralen (IPRN), one of the active ingredients of Psoralea corylifolia Linn, has anti-inflammatory properties. We attempted to investigate the inhibitory effects of IPRN on rheumatoid arthritis (RA) and characterize its potential mechanism.
\end{abstract}

Methods: RA fibroblast-like synoviocytes (FLSs) and mice with collagen-induced arthritis (CIA) were used as in vitro and in vivo models to analyze the antiarthritic effect of IPRN. Histological analysis of the inflamed joints from mice with CIA was performed using microcomputed tomography (micro-CT) and hematoxylin-eosin (HE) staining. RNA sequencing (RNA-Seq), network pharmacology analysis, molecular docking, drug affinity responsive target stability (DARTS) assay, and cellular thermal shift assay (CETSA) were performed to evaluate the targets of IPRN.

Results: IPRN ameliorated the inflammatory phenotype of RA FLSs by inhibiting their cytokine production, migration, invasion, and proangiogenic ability. IPRN also significantly reduced the severity of CIA in mice by decreasing paw thickness, arthritis score, bone damage, and serum inflammatory cytokine levels. A mechanistic study demonstrated that macrophage migration inhibitory factor (MIF), a key protein in the inflammatory process, was the specific target by which IPRN exerted its anti-inflammatory effects in RA FLSs.

Conclusion: Our study demonstrates the antiarthritic effect of IPRN, which suggests the therapeutic potential of IPRN in RA.

Keywords: Rheumatoid arthritis, Isopsoralen, MIF, Collagen-induced arthritis, RA FLSs

\section{Introduction}

Rheumatoid arthritis (RA) is a chronic, autoimmune inflammatory disease characterized by synovial hyperplasia and joint destruction [1]. Persistent inflammation caused by the excessive production of inflammatory cytokines, such as tumor necrosis factor- $\alpha$ (TNF- $\alpha)$, interleukin (IL)-1, IL-6, IL-17, and macrophage-stimulating factor (M-CSF), by fibroblast-like synoviocytes (FLSs), as well as recruited macrophages and immune cells, promotes

\footnotetext{
*Correspondence: geluna606@163.com; kecheng1216@163.com ${ }^{5}$ Biomedical Sciences College \& Shandong Medicinal Biotechnology Centre, Key lab for Biotech-Drugs of National Health Commission, Key Lab for Rare \& Uncommon Diseases of Shandong Province, Shandong First Medical University \& Shandong Academy of Medical Sciences, Jinan, China Full list of author information is available at the end of the article
}

the formation of pannus and consequently destroys the articular cartilage and bone [2, 3]. RA FLSs exhibit tumor-like properties, including enhanced proliferation, prolonged survival, and apoptosis resistance [4, 5]. In addition, RA FLSs can secrete many proinflammatory factors, thereby recruiting inflammatory cells (including macrophages and lymphocytes), inhibiting their apoptosis $[6,7]$, and ultimately promoting local and systemic inflammation. More importantly, RA FLSs produce excessive amounts of matrix metalloproteinases (MMPs), which directly invade and destroy the adjacent articular cartilage [8]. Thus, targeting RA FLSs represents a potential strategy for RA treatment. 
Traditional Chinese medicine (TCM) has long been applied to treat chronic inflammatory diseases and shows efficacy in RA $[9,10]$. In recent years, numerous studies have been carried out to study the anti-RA effect of ingredients from TCM [11]. To date, botanical medicines, such as sinomenine [12], tripterygium glycosides [13], and total glucosides of peony [14], have been developed and applied as alternative treatments for RA. Isopsoralen (IPRN, 2H-Furo[2,3-H] chromen-2-one, CAS Number: 523-50-2, Fig 3b), one of the main ingredients in the seeds of the Psoralea corylifolia Linn plant, has been reported to exert anti-inflammatory effects in inflammatory respiratory and neurodegenerative diseases $[15,16]$. In this study, we aimed to investigate the therapeutic potential of IPRN in RA and the molecular mechanism.

\section{Methods}

\section{Patients and preparation of RA FLSs}

Synovial tissues were obtained from 10 patients who underwent joint replacement surgery at Shandong Provincial Hospital. All the patients were diagnosed according to the 1987 American College of Rheumatology (ACR) revised classification criteria [17] or the European League against Rheumatology (EULAR) classification criteria for RA [18]. This study was approved by the Medical Ethics Committee of the Institutional Review Board of Shandong Medicinal Biotechnology Center (SMBC2020-07), and all the patients signed informed consent forms.

RA FLSs were isolated by the modified tissue culture method we previously described [19]. The cells were cultured in Dulbecco's modified Eagle's medium (DMEM, Hyclone, Logan, UT, USA) supplemented with $10 \%$ fetal bovine serum (FBS) and 1\% antibiotics. RA FLSs from passages 4 6 were used in our experiments.

\section{Migration, invasion, and tube formation assays}

RA FLSs were pretreated with or without TNF- $\alpha$ (Novoprotein, Shanghai, China) in the presence or absence of IPRN (MedChemExpress, Monmouth Junction, NJ, USA) for $24 \mathrm{~h}$. The cell supernatants were collected as a conditioned medium and cultured with HUVECs seeded in 48-well plates for tube formation assays. Migration and invasion assays were performed using Transwell chambers with $8-\mu \mathrm{m}$ pores (Corning, Tewksbury, MA, USA) as previously described [19]. For the invasion assay, Matrigel (Corning, Tewksbury, MA, USA) was precoated on the membrane of the upper chamber, and the cells were seeded as in the migration assay and allowed to invade for $24 \mathrm{~h}$. For the tube formation assay, Matrigel was precoated on 48 -well plates. The data are presented as the mean number of migrated cells in six randomly chosen fields.

\section{Quantitative real-time PCR (qRT-PCR) and enzyme-linked} immunosorbent assay (ELISA)

Total RNA was isolated from RA FLSs using TRIzol Reagent (Invitrogen, Carlsbad, CA, USA) according to the manufacturer's protocol. RNA was reverse-transcribed using a ReverTra Ace qPCR RT Kit (Toyobo, Shanghai, China), and qRT-PCR was performed using a LightCycler 480 (Roche, Basel, Switzerland). All the primers were synthesized by Beijing Genomics Institute (BGI, Beijing, China), and the sequences of all the primers used in this study are listed in Supplied Table 1. Relative mRNA levels were measured using the $2^{-\Delta}$ Cycle Threshold $\left(2^{-\Delta \mathrm{CT}}\right)$ method [20]. Three independent experiments were performed, and each reaction was repeated three times.

The levels of IL-1 $\beta$, IL- 6 , cartilage oligomeric matrix protein (COMP), and IL-10 in the supernatant were determined using commercial ELISA kits (Multi Sciences (Lianke) Biotech Co., Ltd., Hangzhou, China) according to the manufacturer's instructions.

\section{Small interfering RNA transfection and RNA sequencing} The negative control (NC) siRNA and siRNA targeting macrophage migration inhibitory factor (MIF) were synthesized by Ruibo Co., Ltd. (Guangzhou, China). siRNA targeting MIF or NC siRNA was transfected into RA FLSs using transfection reagent (PolyPlus Transfection, Strasbourg, France) according to the manufacturer's instructions. Total RNA was extracted by TRIzol to conduct RT-qPCR or cDNA library construction for RNA transcriptome sequencing by LC-BIO Technologies Co., Ltd. (Hangzhou, China).

\section{Drug affinity responsive target stability (DARTS) assay,} cellular thermal shift assay (CETSA), and Western blotting analysis

DARTS assay [21], CETSA assay [22], and Western blotting analysis were conducted as previously described. Protein samples were separated by SDS-PAGE and electrophoretically transferred onto $0.45-\mu \mathrm{m}$ polyvinylidene fluoride (PVDF) membranes (Millipore, Bedford, MA, USA). The PVDF membranes were blocked and then incubated at $4^{\circ} \mathrm{C}$ overnight with primary antibodies against MIF (Abclonal, Wuhan, China) and glyceraldehyde-3phosphate dehydrogenase (GAPDH) (Proteintech, Wuhan, China). Subsequently, the membranes were washed three times with tris-buffered saline with Tween (TBST) and incubated with HRP-conjugated AffiniPure goat anti-rabbit IgG $(\mathrm{H}+\mathrm{L})$ (Proteintech, Wuhan, China) for 1 $\mathrm{h}$ at room temperature followed by three washes with TBST. The blots were visualized using an enhanced chemiluminescent substrate (ECL) kit (Vazyme, Nanjing, China). The protein bands were quantified using ImageJ software 2.0 (National Institutes of Health, Bethesda, 
Maryland, USA) and were normalized to the density of the respective GAPDH band.

\section{Network pharmacology analysis, molecular docking, and MIF tautomerase assay}

Network pharmacology was used to predict targets as previously described [23]. PharmMapper (http://lilabecust.cn/pharmmapper/), STITCH (http://stitch.embl. de/), and TargetNet (http://targetnet.scbdd.com/calcnet/ index/) were used to predict the targets of IPRN. Duplicates were deleted after combining the targets of IPRN. Targets relevant to rheumatoid arthritis were identified by searching the NCBI gene database with the following keyword: "rheumatoid arthritis." Molecular docking was performed on the Yinfo Cloud Computing Platform (https://cloud.yinfotek.com). L-Dopachrome substrate was prepared through oxidation of L-3,4-dihydroxyphenylalanine methyl ester (Aladdin, Shanghai, China) with sodium periodate (Aladdin, Shanghai, China) [24]. Recombinant MIF (Abcam, Cambridge, MA, USA) (100 $\mathrm{ng} / \mathrm{mL}$ ) was incubated with IPRN or the MIF inhibitor ISO-1 (Selleck, Shanghai, China) at the indicated final concentrations [25]. The decrease in absorbance was measured at $475 \mathrm{~nm}$ every $10 \mathrm{~s}$ for $6 \mathrm{~min}$.

\section{ClA model}

All the animal studies were approved by the Institutional Animal Care and Use Committee of Shandong First Medical University \& Shandong Academy of Medical Sciences. Eight-week-old male DBA/1J mice purchased from Vital River Laboratory Animal Technology (Beijing, China) were raised under specific pathogen-free conditions and were given free access to a standard diet and water. CIA models were established as previously described [19]. Briefly, CIA was induced in mice by injecting $2 \mathrm{mg} / \mathrm{mL}$ bovine type II collagen (Chondrex, Redmond, WA, USA) and complete Freud's adjuvant (Chondrex, Redmond, WA, USA) in a 1:1 emulsion (200 $\mu \mathrm{L})$ at the base of the tail. Booster immunization with a 1:1 emulsion of bovine type II collagen and incomplete Freud's adjuvant $(200 \mu \mathrm{L})$ was administered 21 days after the first immunization. In the therapeutic treatment study, when the arthritis score reached 6 , the mice were randomly divided into groups. IPRN or vehicle (9 mice per group) was administered by intraperitoneal injection into the mice twice a day beginning on the day the mice were divided into groups. The progression of arthritis was continuously monitored after the first immunization by assessing the arthritis score (the sum of scores on a 0-4 scale for all 4 limbs, based on the swelling of the limbs, for a total possible score of 16 for each mouse) using previously described methods [26]. The arthritis score for each mouse was defined as the sum of the scores of all four paws. In each category, the joints were independently evaluated in a blinded manner. In addition, the thickness of the arthritic hind paws was measured daily with microcalipers.

\section{Micro-computed tomography (micro-CT)}

Micro-CT (QuantumGX, PerkinElmer, United States) was performed on the hind paws of mice with CIA with the following parameters: exposure time of $14 \mathrm{~min}$ at 90 $\mathrm{KV}$ and $88 \mathrm{~mA}$ with a resolution of $2 \mu \mathrm{m}$ and field-ofview $12.8 \mathrm{~mm} \times 12.8 \mathrm{~mm}$. The parameters of the trabecular bone, including bone volume to bone volume fraction (BV/TV, \%), trabecular thickness (Tb.Th, $\mu \mathrm{m})$, trabecular bone mineral density (tb.BMD, $\mathrm{g} / \mathrm{cm}^{3}$ ), trabecular number (Tb.N, mm/1), and trabecular separation (Tb.Sp, $\mathrm{mm}$ ) were exported for reconstruction using the manufacturer's software, Caliper Analyze.

\section{Hematoxylin-eosin (H\&E) staining and histopathological examination}

Left proximal tibia joints together with the synovial tissues were fixed in $4 \%$ paraformaldehyde and then decalcified in 10\% EDTA for approximately 3 weeks. The tissues were embedded in paraffin and stained with $\mathrm{H} \& \mathrm{E}$ for light microscopy analysis. Histopathological quantification was evaluated by three blinded, independent pathologists. Inflammation was scored $0-5$ according to the following criteria: 0 , normal; 1 , minimal inflammatory infiltration; 2 , mild infiltration; 3 , moderate infiltration with moderate edema; 4, marked infiltration with marked edema; and 5, severe infiltration with edema. Hyperplasia was defined as synovial tissue intimately invading the bone and/or cartilage and was scored 0 to 3 as follows: 0 , none; 1 , minimal; 2 , moderate; and 3 , severe. Bone degradation was scored using the following 0 to 3 scales: 0 , no bone erosion; 1 , mild surface erosion; 2 , moderate surface erosion; and 3 , strong surface erosion.

\section{Statistical analysis}

Statistical analyses were performed using GraphPad Prism 7 Software (GraphPad Software, Inc., San Diego, CA, USA). The data are expressed as the mean \pm standard deviation (SD). For parametric data, comparisons of different groups were performed by one-way analysis of variance, followed by Tukey's post hoc test for multiple comparisons. $p<0.05$ was considered statistically significant.

\section{Results}

IPRN ameliorates the inflammatory phenotype of RA FLSs RA FLSs display an inflammatory phenotype characterized by the excessive production of inflammatory factors and the enhancement of invasive ability. First, we analyzed the effect of IPRN on cytokine production by RA 
FLSs exposed to TNF- $\alpha$. RT-qPCR and ELISA analysis showed that the induction of IL-6, IL-8, MMP1, MMP3, (C-X-C motif) ligand (CXCL)9, and CXCL10 production resulting from TNF- $\alpha$ stimulation could be significantly attenuated by IPRN treatment (Fig. S1, Fig. 1a). Furthermore, following IPRN treatment, the numbers of migrated and invaded RA FLSs notably decreased in a dose-dependent manner (Fig. 1b, c). We also determined the effect of IPRN on the pro-angiogenesis properties of RA FLSs, and the results showed that after exposure to supernatants from IPRN-treated RA FLSs, the angiogenic ability of HUVECs significantly decreased when compared with that of the parental control cells (Fig. 1d). Thus, the above experiments confirmed that IPRN could ameliorate the inflammatory phenotype of RA FLSs.

\section{IPRN exerts anti-arthritis effects on a CIA model}

The CIA mouse model was then used to investigate the therapeutic potential of IPRN in RA. The results showed that IPRN could inhibit the pathological progression of the RA-like phenotype, as evidenced by the decreased degree of ankle swelling (Fig. 2a), paw thickness (Fig. 2b), and arthritis score (Fig. 2c), compared to the vehicle treatment. We also used micro-CT to quantify the changes in bone destruction in the mice with CIA. Apparently, the animals in the IPRN-treated group suffered less bone damage in their ankle joints than those in the vehicle-treated group, which was shown by the increase in trabecular BV/TV, BMD, Tb.N, and Tb.Th but the decrease in Tb.Sp (Fig. 2d).

HE staining was then conducted to analyze the effect of IPRN on the pathological characteristics of inflamed joints in mice with CIA treated with or without IPRN. As expected, IPRN ameliorated the pathological characteristics of RA, such as synovial hyperplasia, inflammatory cell infiltration, and cartilage damage (Fig. 2e). The serum levels of proinflammatory cytokines (IL-6 and IL$1 \beta$ ) and anti-inflammatory cytokines (IL-10) were also detected in the mice with CIA. IPRN significantly reduced the production of IL-6, IL-1 $\beta$, and COMP but increased the production of IL-10 (Fig. 2f). These results further support the potential efficacy of IPRN in RA treatment.

\section{Prediction of the molecular pathways affected by IPRN in RA FLSs by RNA-Seq}

To further support the anti-inflammatory role of IPRN in RA, RNA-Seq was performed in RA FLSs treated with or without IPRN. A total of 586 differentially expressed genes (DEGs) were observed when RA FLSs were treated with IPRN compared to the control treatment (Fig. 3a). Among these genes, 169 DEGs were upregulated, while 417 were downregulated. A heatmap of the top 20 DEGs is displayed in Fig. 3b. To acquire a more comprehensive and deep understanding of the DEGs, Gene Ontology (GO) function and Kyoto Encyclopedia of Genes and Genomes (KEGG) pathway enrichment analyses were performed. The annotated results for the GO terms were divided into the biological process (BP), cell component (CC), and molecular function (MF) ontologies $(p<0.05$, FDR < 0.05) (Fig. 3c). The results of the GO analysis based on BP revealed that DEGs were mainly enriched in signal transduction, immune system process, inflammatory response, and immune response. For the GO analysis focusing on CC, DEGs were significantly enriched in membrane, cytoplasm, nucleus, and integral component of membrane. In addition, the MFs of the DEGs were mainly enriched in protein binding and metal ion binding.

Among the deregulated pathways, the top 5 downregulated KEGG pathways were as follows: cytokine-cytokine receptor interaction (ko04060), IL-17 signaling pathway (ko04657), NOD-like receptor signaling pathway (ko04621), TNF signaling pathway (ko04668), and rheumatoid arthritis (ko05323) (Fig. 3d). Importantly, these pathways play important roles in the progression of inflammatory diseases, including rheumatic diseases $[27,28]$. Furthermore, RT-qPCR was used to confirm the expression of DEGs with significant roles in the dysregulated KEGG pathways mentioned above (Fig. 3e), and the results confirmed the reliability of the RNA-seq data. Together, IPRN plays an anti-inflammatory role in RA by suppressing the activities of multiple inflammatory pathways.

\section{Identifying MIF as IPRN's direct target}

To identify the molecular targets of IPRN, we applied network pharmacology to conduct target prediction analysis. Target prediction was carried out by online tools, such as PharmMapper and STITCH, and a total of 36 targets were identified. GO annotation showed that some targets, such as a disintegrin and metallopeptidase domain (ADAM17), receptor-interacting serine/threonine-protein kinase 2 (RIPK2), Toll-like receptor 9 (TLR9), RAR-related orphan receptor alpha (RORA), MIF, and aldose reductase (AKR1B1), were closely related to inflammation (GO 0001819, GO 0007249, GO 0043122, GO 0051092) (Fig. 4a). Among these inflammation-related targets, ADAM17, TLR9, MIF, nuclear factor kappaB subunit 1 (NFKB1), NF-kappaB subunit (RELA), and Caspase 8 (CASP8) have been shown to promote the disease progression of RA. As MIF was the target with the highest score, we selected MIF for further study.

Human MIF has tautomerase activity, and residues Lys32, Ile64, Val106, and Phe113 are located at the rim of the active sites of the MIF protein [29]. Then, 


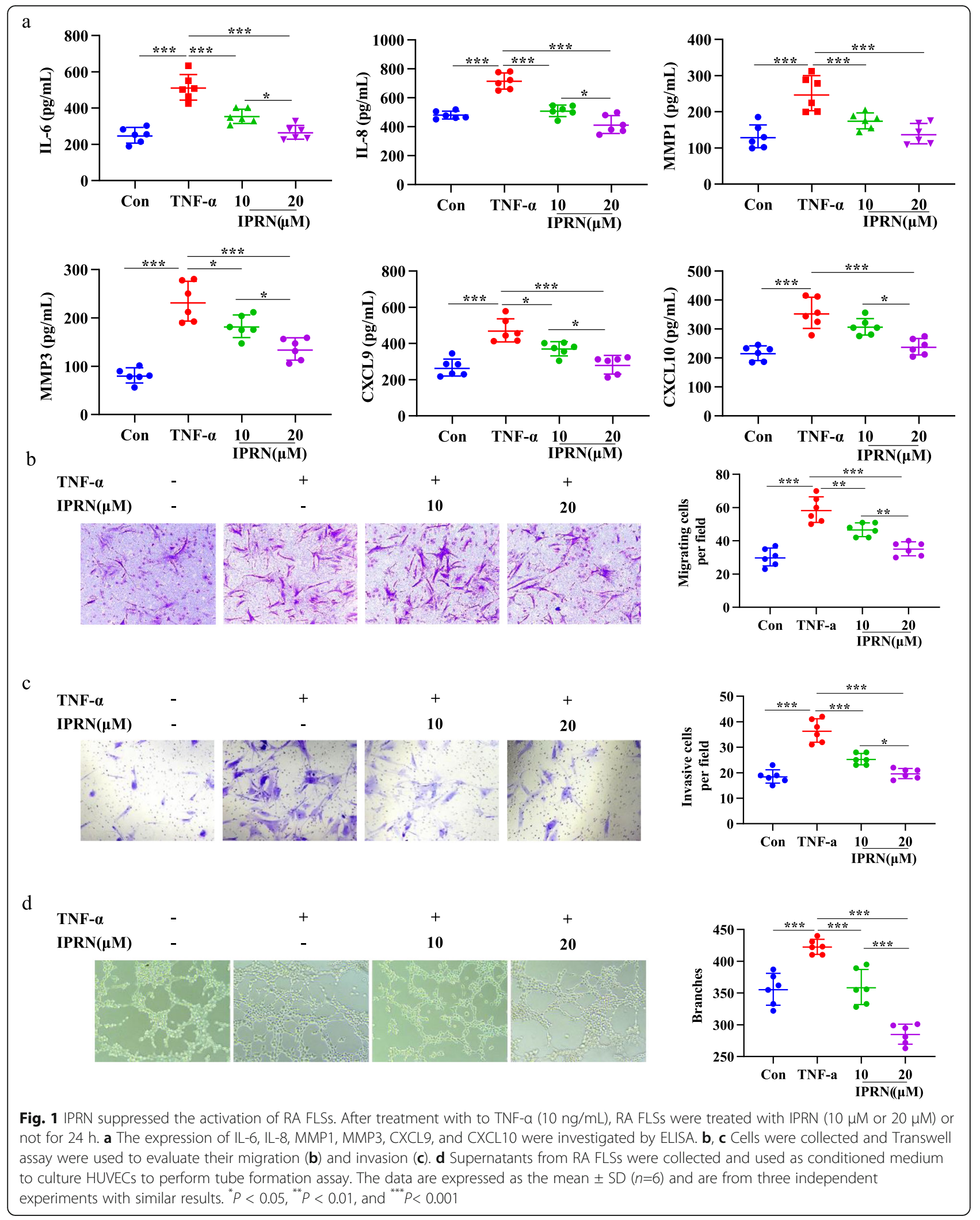






molecular docking of IPRN with MIF (PDB ID:1GCZ) was carried out using DOCK6, and their potential interacting sites are presented in Fig. 4b. Interestingly, IPRN showed a hydrogen bond interaction with residues Lys
32 and Ile 64 of the MIF protein. In addition, IPRN formed hydrophobic interactions with the residues His62, Val 106, and Phe113. We also detected the effect of IPRN on the tautomerase activity of MIF. As 


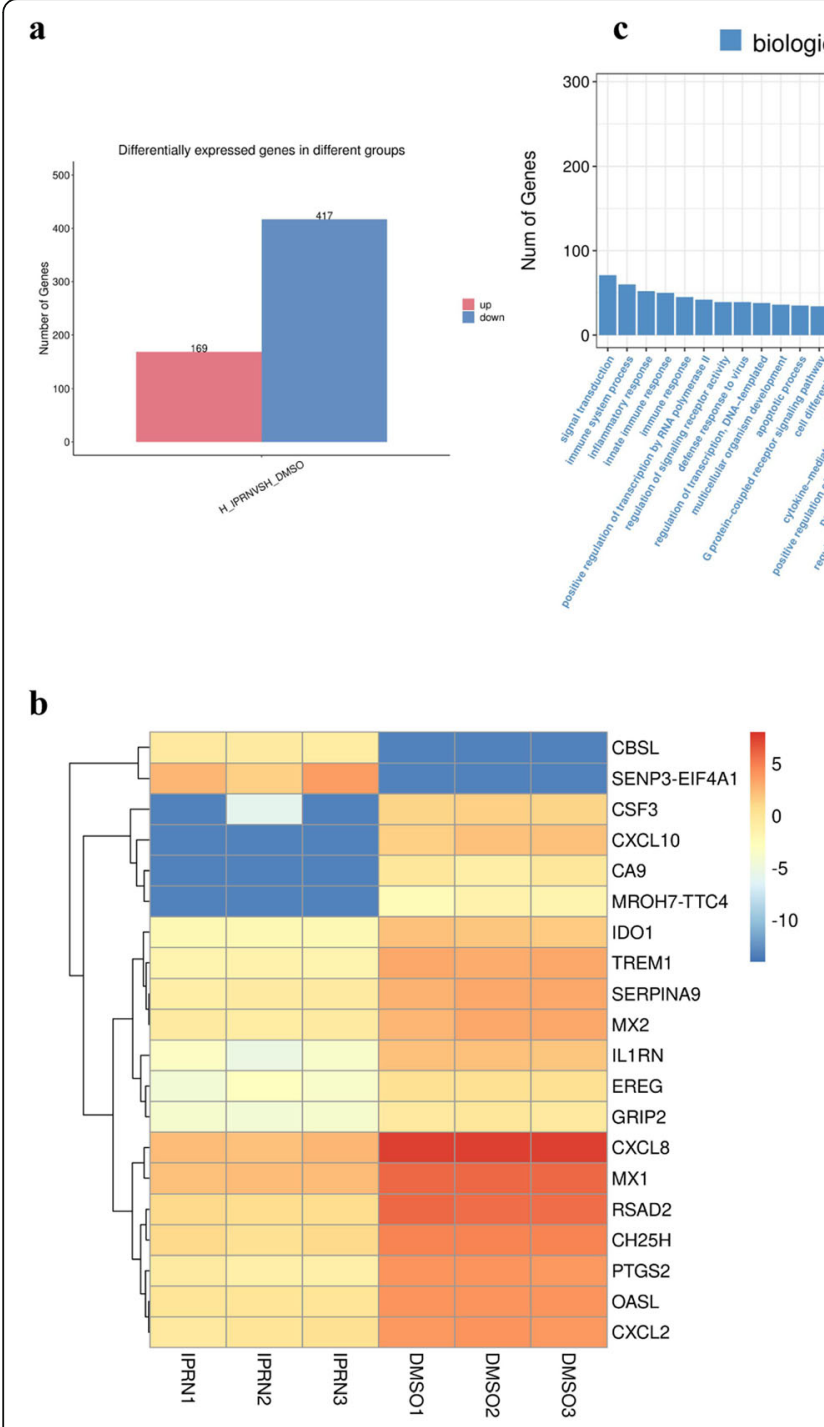

d

e



Fig. 3 Molecular pathway prediction of the antiarthritic effect of IPRN in RA FLSs by RNA-Seq. RA FLSs were treated without or with IPRN (10 $\mu M$ ) in the presence of TNF-a $(10 \mathrm{ng} / \mathrm{mL})$ for $24 \mathrm{~h}$. Total RNA was extracted for RNA-seq. a The number of differentially expressed genes (DEGs) induced by IPRN. $\mathbf{b}$ Heatmap displays different blocks of top 20 DEGs between the IPRN-treated cells $(n=3)$ and the control cells $(n=3)$. $\mathbf{c}$ The GO analysis of DEGs induced by IPRN. Bar plots show the significant enrichment terms of the DEGs involving biological process, cellular component, and molecular function ranked by enrichment score ( $-\log 10$ ( $p$ value)) values. d KEGG enrichment of DEGs. e Verification of DEGs by RT-qPCR. The data are expressed as the mean \pm SD $(n=6) .{ }^{*} P<0.05,{ }^{* * * *} P<0.001$ 


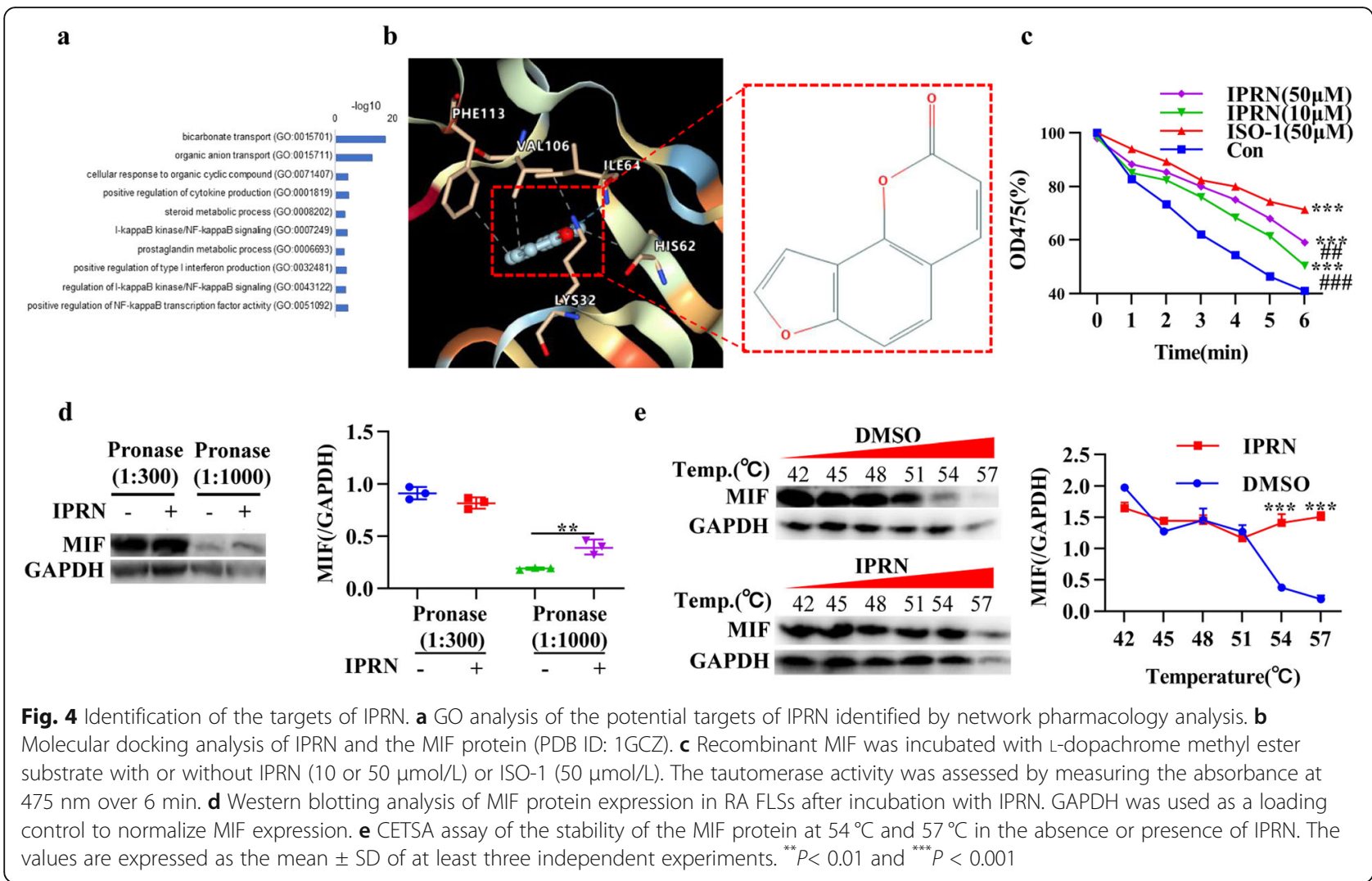

expected, IPRN significantly inhibited the enzyme activity of recombinant human MIF (Fig. 4c). Moreover, the activity of MIF in RA FLSs and serum obtained from mice with CIA treated with IPRN decreased (Fig. S2d, e).

To verify the interaction of IPRN with MIF, DARTS and CETSA experiments were conducted. The DARTS results showed that IPRN can protect the degradation of the MIF protein caused by proteases (Fig. 4d). In addition, the CETSA assay showed that IPRN significantly improved the stability of MIF at $54^{\circ} \mathrm{C}$ and $57^{\circ} \mathrm{C}$ (Fig. 4e). However, IPRN did not affect the expression of MIF in RA FLSs (Fig. S2a-c).

In conclusion, the results described above indicate that MIF is a potential target of IPRN and that IPRN may target and inactivate MIF.

\section{IPRN inhibits inflammation via MIF}

MIF acts as an important contributor to the pathological progression of RA. Consistently, following treatment with recombinant human MIF, the expression of IL-6, IL-8, MMP3, and CXCL9 significantly increased. However, this increase could be attenuated when RA FLSs were simultaneously treated with IPRN (Fig. 5a). To further confirm the importance of MIF for the antiinflammatory effect of IPRN, siRNA targeting MIF was introduced. After knocking down MIF expression in
RAFLSs, the inhibitory effects of IPRN on TNF- $\alpha$ induced cytokine release (Fig. 5b) and the proangiogenic ability (Fig. 5c) of RA FLSs could hardly be observed. These data suggest that MIF acts as an essential contributor to the anti-inflammatory effect of IPRN.

\section{Discussion}

The application of disease-modifying anti-rheumatic drugs (DMARDs) and biological agents has improved the therapeutic effects in RA [30]. However, these treatment strategies still have some problems, especially the increased susceptibility to infection caused by long-term treatment or the existence of refractory rheumatoid arthritis patients who do not respond well to these existing medicines [31, 32]. Recently, complementary and alternative medicine approaches including TCM have been shown to increase the likelihood of complete remission of RA [32, 33]. More detailed studies concerning the antiarthritic mechanism of TCMs or their natural ingredients may provide novel treatment strategies for RA.

Previous studies on IPRN have mainly focused on its antibacterial, osteogenic, and anti-inflammatory effects. Following treatment of ovalbumin-induced lung injury models with IPRN, the infiltration of inflammatory cells and the production of inflammatory factors were suppressed [34]. Furthermore, IPRN prevented the alveolar bone loss and obviously inactivated the inflammatory 


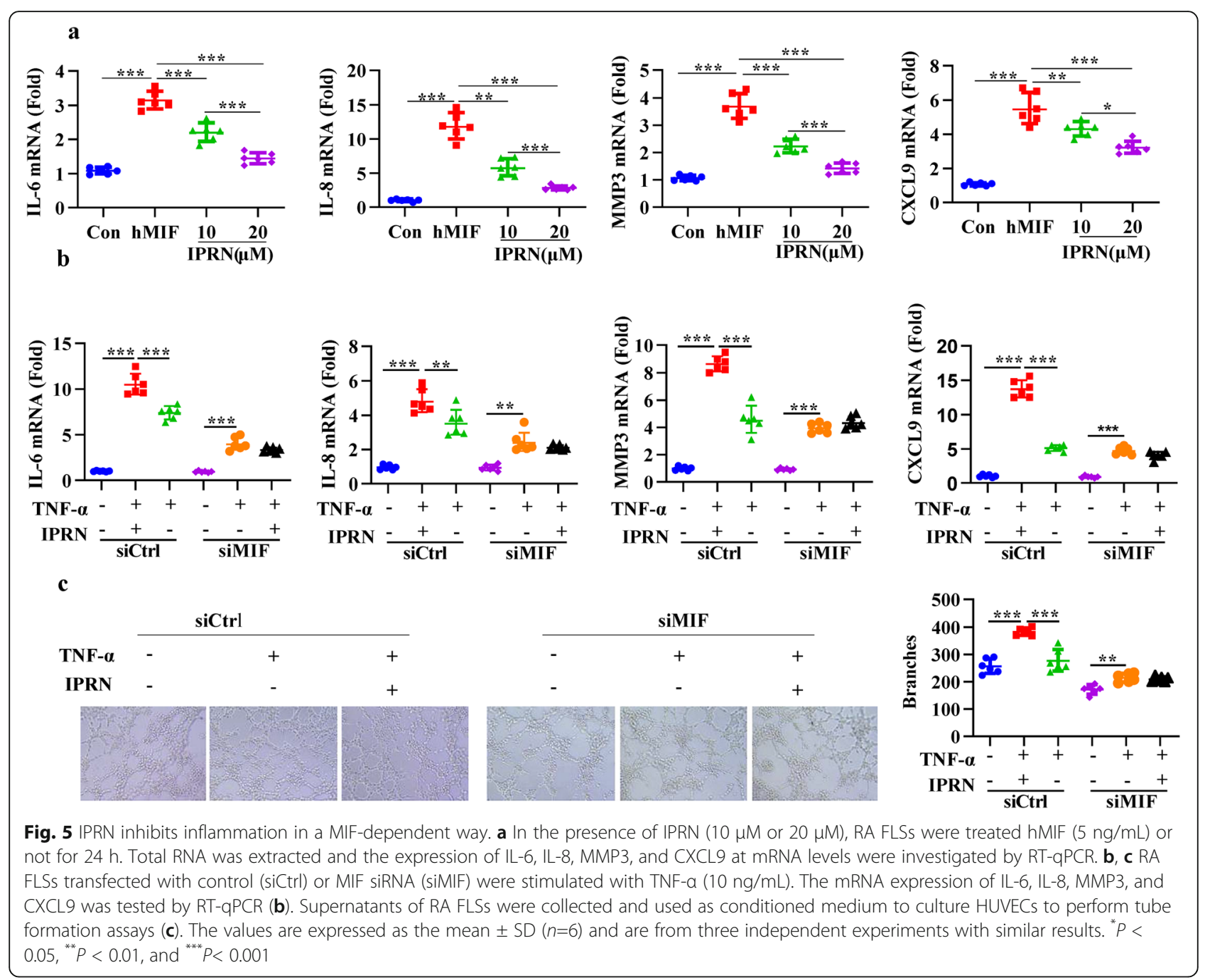

response in mice with periodontitis [15]. Mechanistically, IPRN protects against the inflammation caused by lipopolysaccharide (LPS) challenge in both in vitro and in vivo models by inhibiting the activation of the NF- $\mathrm{kB}$ and mitogen-activated protein kinase (MAPK) pathways [35]. In this study, we characterized the antiarthritic effect of IPRN in both cultured human RA FLSs and a CIA model, and IPRN represents a potential drug for RA treatment that can inhibit the inflammatory phenotype of RA FLSs and prevent the onset and progression of RA-like manifestations in a CIA model.

MIF acts as a pleiotropic inflammatory cytokine that is important in both innate and adaptive immunological responses and has been implicated in the pathogenesis of autoimmune diseases, including RA and systemic lupus erythematosus (SLE) [36]. Elevated levels of MIF expression were observed in synovial tissues and synovial fluids from RA patients compared to osteoarthritis (OA) patients or healthy volunteers [37]. Specifically, the expression of MIF was closely related to the activity of RA, and its expression at higher levels indicated more severe bone erosion [38]. Functionally, MIF was required for sustaining inflammatory responses by inducing the production of proinflammatory cytokines and tissuedegrading molecules, promoting the proliferation and survival of synovial fibroblasts, stimulating the chemotaxis of neutrophils, and increasing angiogenesis and osteoclast differentiation. In animal models of RA, the genetic and therapeutic inhibition of MIF has been shown to suppress inflammation and ameliorate bone destruction [39, 40]. Furthermore, MIF-targeted treatments are being examined in preclinical studies or earlystage clinical trials due to their specific functions observed in different autoimmune diseases. The development of small-molecule MIF antagonists could provide a means of selectively intervening in the pathogenesis of RA [40, 41]. Here, our data confirmed that IPRN targeted and inactivated MIF and that MIF was required 
for the anti-inflammatory effect of IPRN, further confirming the likelihood that IPRN could be further exploited as a drug for RA treatment.

\section{Conclusion}

In summary, the results from our in vivo and in vitro experiments support the antiarthritic effects of IPRN in RA, and MIF represents a target by which IPRN exerts its anti-inflammatory effects. Therefore, IPRN holds great potential as a novel therapeutic agent for RA.

\begin{abstract}
Abbreviations
RA: Rheumatoid arthritis; micro-CT: Computed tomography; TNF-a: Tumor necrosis factor-a; ILs: Interleukins; FLSs: fibroblast-like synoviocytes; MMP: Matrix metalloproteinase; TCM: Traditional Chinese medicine; IPRN: Isopsoralen; CIA: Collagen-induced arthritis; MIF: Macrophage migration inhibitory factor; GAPDH: Glyceraldehyde-3-phosphate dehydrogenase; TBST: Tris-buffered saline with tween; RNA-Seq: RNA sequencing: DARTS: Drug affinity responsive target stability; CETSA: Cellular thermal shift assay; CXCL: (C-X-C motif) ligand; BV/TV: Bone volume/total volume; BMD: Bone mineral density; Tb.N: Trabecular number; Tb.Th: Trabecular thickness; Tb.Sp: Trabecular separation; COMP: Cartilage oligomeric matrix protein; DEGs: Differentially expressed genes; GO: Gene ontology; KEGG: Kyoto Encyclopedia of Genes and Genomes; ADAM17: A disintegrin and metallopeptidase domain 17; RIPK2: Receptor-interacting serine/ threonine-protein kinase 2; TLR9: Toll-like receptor 9; RORA: RAR-related orphan receptor alpha; AKR1B1: Aldose reductase; DMARDs: Diseasemodifying anti-rheumatic drugs; NF-kB: Nuclear factor-kappaB; MAPK: Mitogen-activated protein kinase; NFKB1: Nuclear factor kappaB subunit 1; RELA: NF-kappaB subunit; CASP8: Caspase 8
\end{abstract}

\section{Supplementary Information}

The online version contains supplementary material available at https://doi. org/10.1186/s13075-021-02619-3.

\section{Additional file 1: Figure S1.}

Additional file 2: Figure S2.

Additional file 3: Supplied Table 1.

\section{Acknowledgements}

Not applicable

\section{Authors' contributions}

LW and LG conceived the study. YH, YZ, RZ, and YZ performed the cell studies. YH, JW, YZ, and HF performed the animal experiments. JP and GS performed the statistical analysis. YH and LG analyzed the data and wrote the manuscript. The authors discussed the results and commented on the manuscript. The author(s) read and approved the final manuscript.

\section{Funding}

This work was supported by the National Natural Science Foundation of China (Grant No. 81772760, 81901666, 82072850), Taishan Scholar Project of Shandong Province (No. tsqn20161076), The Innovation Project of Shandong Academy of Medical Sciences, The Youth Innovation Technology Plan of Shandong University (2019KJK003), and Academic Promotion Programme of Shandong First Medical University (เ001).

\section{Availability of data and materials}

The authors are committed to sharing their data, publishing the data, and making available the resources described in this publication to the scientific community.

\section{Declarations}

Ethics approval and consent to participate

De-identified human tissues were collected after approval of the institutional review board (IRB). Animal studies were performed under institutional guidelines and following protocols approved by the Institutional Animal Care and Use Committee (IACUC).

\section{Consent for publication}

Not applicable

\section{Competing interests}

The authors declare that they have no competing interests.

\section{Author details}

'China Academy of Chinese Medical Sciences, Guang'anmen Hospital, Beijing, China. 'Department of Orthopedics, Dezhou People's Hospital, Dezhou, Shandong, China. ${ }^{3}$ Department of Orthopedic Surgery, The First Affiliated Hospital of Shandong First Medical University, Jinan, China. ${ }^{4}$ Department of Joint Surgery, Shandong Provincial Hospital Affiliated to Shandong University, Jinan, China. ${ }^{5}$ Biomedical Sciences College \& Shandong Medicinal Biotechnology Centre, Key lab for Biotech-Drugs of National Health Commission, Key Lab for Rare \& Uncommon Diseases of Shandong Province, Shandong First Medical University \& Shandong Academy of Medical Sciences, Jinan, China. ${ }^{6}$ Department of Rheumatology and Autoimmunology, The First Affiliated Hospital of Shandong First Medical University, Jinan, China. 'Biomedical Sciences College \& Shandong Medicinal Biotechnology Center, Key lab for Biotech-Drugs of National Health Commission, Key Lab for Rare \& Uncommon Diseases of Shandong Province, Shandong First Medical University \& Shandong Academy of Medical Sciences, \#18877, Jingshi Road, Jinan 250062, China. ${ }^{8}$ The Second Clinical Medical College, Henan University of Chinese Medicine, Jinan, China. ${ }^{9}$ Institute of Basic Medicine, Shandong First Medical University \& Shandong Academy of Medical Sciences, Jinan, China.

Received: 22 December 2020 Accepted: 27 August 2021

Published online: 17 September 2021

\section{References}

1. Nerurkar L, Siebert S, McInnes IB, Cavanagh J. Rheumatoid arthritis and depression: an inflammatory perspective. Lancet Psychiatry. 2019;6(2):16473. https://doi.org/10.1016/s2215-0366(18)30255-4.

2. Korb-Pap A, Stratis A, Mühlenberg K, Niederreiter B, Hayer S, Echtermeyer F, et al. Early structural changes in cartilage and bone are required for the attachment and invasion of inflamed synovial tissue during destructive inflammatory arthritis. Ann Rheum Dis. 2012;71(6):1004-11. https://doi.org/1 0.1136/annrheumdis-2011-200386.

3. Schett G, Gravallese E. Bone erosion in rheumatoid arthritis: mechanisms, diagnosis and treatment. Nat Rev Rheumatol. 2012;8(11):656-64. https://doi. org/10.1038/nrrheum.2012.153.

4. You S, Koh JH, Leng L, Kim WU, Bucala R. The tumor-like phenotype of rheumatoid synovium: molecular profiling and prospects for precision medicine. Arthritis Rheumatol. 2018;70(5):637-52. https://doi.org/10.1002/a rt.40406.

5. Liu Y, Pan YF, Xue YQ, Fang LK, Guo XH, Guo X, et al. uPAR promotes tumor-like biologic behaviors of fibroblast-like synoviocytes through PI3K Akt signaling pathway in patients with rheumatoid arthritis. Cell Mol Immunol. 2018;15(2):171-81. https://doi.org/10.1038/cmi.2016.60.

6. Zhang LM, Zhou JJ, Luo CL. CYLD suppression enhances the proinflammatory effects and hyperproliferation of rheumatoid arthritis fibroblast-like synoviocytes by enhancing NF-KB activation. Arthritis Res Ther. 2018;20(1):219. https://doi.org/10.1186/s13075-018-1722-9.

7. Liu F, Feng XX, Zhu SL, Huang HY, Chen YD, Pan YF, et al. Sonic Hedgehog signaling pathway mediates proliferation and migration of fibroblast-like synoviocytes in rheumatoid arthritis via MAPK/ERK signaling pathway. Front Immunol. 2018;9:2847. https://doi.org/10.3389/fimmu.2018.02847.

8. Malemud CJ. Matrix metalloproteinases and synovial joint pathology. Prog Mol Biol Transl Sci. 2017;148:305-25. https://doi.org/10.1016/bs.pmbts.2017. 03.003 .

9. Lu MC, Livneh H, Chiu LM, Lai NS, Yeh CC, Tsai TY. A survey of traditional Chinese medicine use among rheumatoid arthritis patients: a claims data- 
based cohort study. Clin Rheumatol. 2019;38(5):1393-400. https://doi.org/1 0.1007/s10067-018-04425-w.

10. Santosa A, Ng PS, Teng GG. Traditional Chinese medication for rheumatoid arthritis: more than what meets the eye. Rheumatol Int. 2015;35(2):383-4. https://doi.org/10.1007/s00296-014-3092-1.

11. Shi Y, Shu H, Wang X, Zhao H, Lu C, Lu A, et al. Potential advantages of bioactive compounds extracted from traditional Chinese medicine to inhibit bone destructions in rheumatoid arthritis. Front Pharmacol. 2020;11:561962. https://doi.org/10.3389/fphar.2020.561962.

12. Liu W, Zhang Y, Zhu W, Ma C, Ruan J, Long H, et al. Sinomenine inhibits the progression of rheumatoid arthritis by regulating the secretion of inflammatory cytokines and monocyte/macrophage subsets. Front Immunol. 2018;9:2228. https://doi.org/10.3389/fimmu.2018.02228.

13. Zhang $Y$, Wang $H$, Mao $X$, Guo Q, Li W, Wang $X$, et al. A novel circulating miRNA-based model predicts the response to tripterysium glycosides tablets: moving toward model-based precision medicine in rheumatoid arthritis. Front Pharmacol. 2018;9:378. https://doi.org/10.3389/fphar.2018.003 78.

14. Zhang L, Wei W. Anti-inflammatory and immunoregulatory effects of paeoniflorin and total glucosides of paeony. Pharmacol Ther. 2020;207: 107452. https://doi.org/10.1016/.jpharmthera.2019.107452.

15. Li X, Yu C, Hu Y, Xia X, Liao Y, Zhang J, et al. New application of psoralen and angelicin on periodontitis with anti-bacterial, anti-inflammatory, and osteogenesis effects. Front Cell Infect Microbiol. 2018;8:178. https://doi.org/1 0.3389/fcimb.2018.00178.

16. Mahendra CK, Tan LTH, Lee WL, Yap WH, Pusparajah P, Low LE, et al. Angelicin-A furocoumarin compound with vast biological potential. Front Pharmacol. 2020;11:366. https://doi.org/10.3389/fphar.2020.00366.

17. Liao KP, Batra KL, Chibnik L, Schur PH, Costenbader KH. Anti-cyclic citrullinated peptide revised criteria for the classification of rheumatoid arthritis. Ann Rheum Dis. 2008;67(11):1557-61. https://doi.org/10.1136/ard.2 007.082339 .

18. Fernández-Codina, A.; Pinilla, B.; Pinal-Fernández, l.; Carballo, l.; Feijoo-Massó, C.; Toledano-Macías, M.; de Miguel-Campo, B.; Fonseca-Aizpuru, E.; SáezComet, L.; López-Dupla, M., et al. Performance of the 2019 ACR/EULAR classification criteria for lgG4-related disease and clinical phenotypes in a Spanish multicentre registry (REERIGG4). Rheumatology (Oxford) 2020 doi: https://doi.org/10.1093/rheumatology/keaa247.

19. Song G, Feng T, Zhao R, Lu Q, Diao Y, Guo Q, et al. CD109 regulates the inflammatory response and is required for the pathogenesis of rheumatoid arthritis. Ann Rheum Dis. 2019;78(12):1632-41. https://doi.org/10.1136/a nnrheumdis-2019-215473.

20. Livak KJ, Schmittgen TD. Analysis of relative gene expression data using real-time quantitative PCR and the 2(-Delta Delta C(T)) Method. Methods. 2001;25(4):402-8. https://doi.org/10.1006/meth.2001.1262.

21. Lomenick B, Hao R, Jonai N, Chin RM, Aghajan M, Warburton S, et al. Target identification using drug affinity responsive target stability (DARTS). Proc Natl Acad Sci U S A. 2009;106(51):21984-9. https://doi.org/10.1073/pnas.091 0040106.

22. Martinez Molina D, Jafari R, Ignatushchenko M, Seki T, Larsson EA, Dan C, et al. Monitoring drug target engagement in cells and tissues using the cellular thermal shift assay. Science. 2013;341(6141):84-7. https://doi.org/1 $0.1126 /$ science. 1233606.

23. Ge L, Cheng K, Han J. A network pharmacology approach for uncovering the osteogenic mechanisms of Psoralea corylifolia Linn. Evid Based Complement Alternat Med. 2019;2019:2160175-10. https://doi.org/10.1155/2 019/2160175.

24. Healy ZR, Liu H, Holtzclaw WD, Talalay P. Inactivation of tautomerase activity of macrophage migration inhibitory factor by sulforaphane: a potential biomarker for anti-inflammatory intervention. Cancer Epidemiol Biomarkers Prev. 2011;20(7):1516-23. https://doi.org/10.1158/1055-9965.epi-11-0279.

25. Al-Abed Y, Dabideen D, Aljabari B, Valster A, Messmer D, Ochani M, et al. ISO-1 binding to the tautomerase active site of MIF inhibits its proinflammatory activity and increases survival in severe sepsis. J Biol Chem. 2005;280(44):36541-4. https://doi.org/10.1074/jbc.C500243200.

26. Brand DD, Latham KA, Rosloniec EF. Collagen-induced arthritis. Nat Protoc. 2007;2(5):1269-75. https://doi.org/10.1038/nprot.2007.173.

27. Vieira SM, Cunha TM, França RF, Pinto LG, Talbot J, Turato WM, et al. Joint NOD2/RIPK2 signaling regulates IL-17 axis and contributes to the development of experimental arthritis. J Immunol. 2012;188(10):5116-22. https://doi.org/10.4049/jimmunol.1004190.
28. Li WC, Bai L, Xu Y, Chen H, Ma R, Hou WB, et al. Identification of differentially expressed genes in synovial tissue of rheumatoid arthritis and osteoarthritis in patients. J Cell Biochem. 2019;120(3):4533-44. https://doi. org/10.1002/jcb.27741

29. Orita M, Yamamoto S, Katayama N, Aoki M, Takayama K, Yamagiwa Y, et al. Coumarin and chromen-4-one analogues as tautomerase inhibitors of macrophage migration inhibitory factor: discovery and X-ray crystallography. J Med Chem. 2001;44(4):540-7. https://doi.org/10.1021/jm000386o.

30. Choy, E.H. Effect of biologics and targeted synthetic disease-modifying antirheumatic drugs on fatigue in rheumatoid arthritis. Rheumatology (Oxford) 2019, 58, v51-v55, doi:https://doi.org/10.1093/rheumatology/kez389, Supplement_5.

31. Buch MH. Defining refractory rheumatoid arthritis. Ann Rheum Dis. 2018; 77(7):966-9. https://doi.org/10.1136/annrheumdis-2017-212862.

32. Keisuke I, Bian BL, Li XD, Takashi S, Akira I. Action mechanisms of complementary and alternative medicine therapies for rheumatoid arthritis. Chin J Integr Med. 2011;17(10):723-30. https://doi.org/10.1007/s11655-0110871-3.

33. Wang, W.; Zhou, H.; Liu, L. The role of Chinese herbal medicine in the management of adverse drug reactions of leflunomide in treating rheumatoid arthritis. Phytomedicine: 2020, 68, 153136, doi:https://doi.org/1 0.1016/j.phymed.2019.153136.

34. Wei $D Z$, Guo $X Y$, Lin $L N$, Lin $M X$, Gong $Y Q$, Ying $B Y$, et al. Effects of angelicin on ovalbumin (OVA)-induced airway inflammation in a mouse model of asthma. Inflammation. 1876-1882;2016(6):39-1882. https://doi.org/10.1007/s1 0753-016-0423-2.

35. Li L, Eun JS, Nepal M, Ryu JH, Cho HK, Choi BY, et al. Isopsoralen induces differentiation of prechondrogenic ATDC5 cells via activation of MAP kinases and BMP-2 signaling pathways. Biomol Ther (Seoul). 2012;20(3):299305. https://doi.org/10.4062/biomolther.2012.20.3.299.

36. Bozza MT, Lintomen L, Kitoko JZ, Paiva CN, Olsen PC. The role of MIF on eosinophil biology and eosinophilic inflammation. Clin Rev Allergy Immunol. 2020;58(1):15-24. https://doi.org/10.1007/s12016-019-08726-z.

37. Liu M, Hu C. Association of MIF in serum and synovial fluid with severity of knee osteoarthritis. Clin Biochem. 2012;45(10-11):737-9. https://doi.org/10.1 016/j.clinbiochem.2012.03.012.

38. Wakabayashi K, Otsuka K, Sato M, Takahashi R, Odai T, Isozaki T, et al. Elevated serum levels of macrophage migration inhibitory factor and their significant correlation with rheumatoid vasculitis disease activity. Mod Rheumatol. 2012;22(1):59-65. https://doi.org/10.1007/s10165-011-0466-Z.

39. Zheng L, Gao J, Jin K, Chen Z, Yu W, Zhu K, et al. Macrophage migration inhibitory factor (MIF) inhibitor 4-IPP suppresses osteoclast formation and promotes osteoblast differentiation through the inhibition of the NF-KB signaling pathway. FASEB J. 2019;33(6):7667-83. https://doi.org/10.1096/fi.2 01802364RR.

40. Kang I, Bucala R. The immunobiology of MIF: function, genetics and prospects for precision medicine. Nat Rev Rheumatol. 2019;15(7):427-37. https://doi.org/10.1038/s41584-019-0238-2.

41. Kok T, Wasiel AA, Cool RH, Melgert BN, Poelarends GJ, Dekker FJ. Smallmolecule inhibitors of macrophage migration inhibitory factor (MIF) as an emerging class of therapeutics for immune disorders. Drug Discov Today. 1910-1918;2018(11):23-1918. https://doi.org/10.1016/j.drudis.2018.06.017.

\section{Publisher's Note}

Springer Nature remains neutral with regard to jurisdictional claims in published maps and institutional affiliations.

\section{Ready to submit your research? Choose BMC and benefit from:}

- fast, convenient online submission

- thorough peer review by experienced researchers in your field

- rapid publication on acceptance

- support for research data, including large and complex data types

- gold Open Access which fosters wider collaboration and increased citations

- maximum visibility for your research: over $100 \mathrm{M}$ website views per year

At BMC, research is always in progress.

Learn more biomedcentral.com/submission 